\title{
Extreme physical exhaustion leading to hypokalemic periodic paralysis- a case report
}

\author{
Monjuri Borkotokey, MD ${ }^{1}$, Kaustuv Dutta, MD, DNB, DM ${ }^{2}$
}

Author affiliations:

1. Assistant Professor, Department of Biochemistry, Lokopriya Gopinath Bordoloi Regional Institute of Mental Health (LGBRIMH), Kalibari Main Road, Mahabhairab, Tezpur, Assam 784001, India.

2. Assistant Professor, Department of Anesthesiology \& Critical Care, North Eastern Indira Gandhi Regional Institute of Health and Medical Sciences (NEIGRIHMS), Mawdiangdiang, Shillong, Meghalaya 793018, India.

Correspondence: Dr. Kaustuv Dutta; E-mail: kaustuv.dutta@gmail.com

\section{Abstract}

Sudden onset limb weakness in a young individual requires a thorough evaluation and prompt diagnosis. Delay in treatment awaiting CT scan reports or laboratory investigations can lead to complications. Our case report stresses upon the clinicians to consider hypokalemic periodic paralysis in the differential diagnosis in evaluating such patients. Classical ECG changes and blood gas analysis can effectively diagnose hypokalemia and institute gradual correction of the electrolyte abnormality. In a very rare incident, our patient developed hypokalemia after strenuous exercise.

Key words: Hypokalemic periodic paralysis; Hypokalemia; Flaccid paralysis; Exhaustion

Citation: Borkotokey M, Dutta K. Extreme physical exhaustion leading to hypokalemic periodic paralysis- a case report. Anaesth. pain intensive care 2022;26(1):123-125. DOI: 10.35975/apic.v26i1.1782

Received: November 30, 2021, Reviewed: December 25, 2021,, Accepted December 27, 2021

\section{Introduction}

We present a case report of one of our radiology technicians, who suffered from flaccid paralysis of his lower limbs and fell down while at work. On extensive work-up, a diagnosis of hypokalemic periodic paralysis was made based on a very low potassium level and by the exclusion of the other possible causes. The diagnosis was further confirmed by a rapid improvement of the patient when potassium was replaced by infusion.

\section{Case report}

A 24-year old radiology technician in the radiology department, while going about his usual duties of operating the $\mathrm{C}$-arm machine in the operating room (OR), suddenly fell on the floor. He was unable to move his lower limbs and had mild weakness in the upper limbs. On examination, the neuro-anesthetist found that the patient had flaccid paralysis of both of his lower limbs. Deep tendon reflexes were absent and the motor power was $0 / 5$ in the lower limbs and $3 / 5$ in the upper limbs. The weakness involved the proximal shoulder girdle and the distal extremities. There was no sensory impairment, no facial deviation, or any other cranial nerve deficits. He had no complaints of pain, tingling, or numbness in the extremities. There was no difficulty in breathing or swallowing and he was able to lift the neck off the ground.

The patient denied any history of similar episodes in the past or recent changes in bowel habits, appetite, weight loss, or palpitations. There was no family history of seizures or thyroid disorders. On further inquiring, he told that he used to play football once in a while; the previous day he played the game without the usual warmup. He had been exhausted by the end of the game. His vital signs were stable and ECG showed $\mathrm{T}$-wave inversion with $\mathrm{U}$-waves in chest leads.

Complete laboratory workup was done, including electrolyte assay, blood glucose, thyroid function tests, $\mathrm{CK}$, and CK-MB. A venous blood gas analysis was done, which showed a potassium level of $2.1 \mathrm{mmol} / \mathrm{L}$ (normal level $=3.5-5.1 \mathrm{mmol} / \mathrm{L})$. The patient was shifted to the Intensive Care Unit (ICU) and intravenous potassium replacement started. The vital signs were continually 
monitored and the motor power, sensory examination, and cranial nerve tests were done periodically. The intensivist ordered a CT scan of the brain to rule out stroke in this young person. CT brain revealed no abnormality. Over the next few hours, the laboratory reports confirmed hypokalemia and the patient showed gradual improvement over the next 24 hours. The vital signs, including blood pressure, pulse rate, peripheral oxygen saturation were stable throughout the ICU stay. The reports of thyroid function tests, CK, and CK-MB were within normal limits. The patient regained his motor power completely and was back on his feet again. He was shifted to the ward; from there he was discharged home a day later.

\section{Discussion}

A young healthcare professional, with no known previous disease, suddenly collapsing in the OR or a classroom, is not a common phenomenon. Once the initial red flags like syncope and hypoglycemia have been ruled out, other possibilities like stroke and electrolyte abnormalities including hypokalemia should be considered. A detailed history with duration and distribution of weakness, and physical examination for the associated neurological deficits is essential to rule out Guillain-Barre syndrome (GBS), Myasthenia Gravis (MG), and motor neuron disease.

Hypokalemic periodic paralysis (HPP) is a rare spectrum of disorders that can cause sudden onset weakness with flaccid paralysis. ${ }^{1}$ The reported prevalence of HPP is 1 in 100,000 with most patients presenting with sudden weakness, which can range from mild to severe disability, with underlying metabolic and electrolyte derangements. $^{2}$ The biochemical mechanism of hypokalemia is due to abnormally functioning sodium and calcium ion channels, resulting in muscle dysfunction. Potassium is a major intracellular cation that plays a vital role in maintaining the physiological functions of various membranes in the body, including the cardiac and skeletal muscles. HPP is a diagnosis of exclusion and requires a thorough workup to search for any provoking factors like stress, viral illness, or thyroid dysfunction. Familial hypokalemic paralysis (FHP) is a genetic disorder with autosomal dominant inheritance due to mutations in CACNA1S and SCN4A genes that cause sodium channel dysfunction leading to the movement of extracellular potassium into the cells. Researchers have identified mutations that cause abnormality in inward rectifier potassium channels ${ }^{4}$ that may be responsible for FHP. All of these mutations result in aberrant passage of ions through the channels, resulting in non-conduction of the action potential and rendering the muscles placid. This phenomenon is responsible for frequent episodes of flaccid paralysis. ${ }^{5}$
Thyrotoxic periodic paralysis (TPP) is the most common form of HPP. The presentation of TPP, besides the sudden onset of weakness, also includes features of hyperthyroidism like sweating, recent weight loss, bowel disturbances, and nervousness. These symptoms could be subtle and often missed by the patient. ${ }^{6}$

HPP resulting from extreme physical exhaustion has rarely been reported in the literature. Our patient had an episode of sudden onset of weakness presumably because of excessive physical activity, owing partly due to skipping the warm-up routine before the football match. As he denied any significant family history and we ruled out all other common causes of flaccid paralysis, we concluded that the patient had HPP. Genetic testing for FHP is not available at our center. The patient is being followed up for the past six months, and there has been no recurrence of similar episodes.

\section{Conclusion}

Hypokalemic periodic paralysis should be considered in the differential diagnosis in the workup of young patients with acute onset weakness with no major sensory symptoms. It may avoid subjecting them to a stroke protocol or ordering a CT scan. Timely recognition of periodic paralysis with gradual potassium replacement can lead to rapid resolution of symptoms. Simple measures like adequate hydration, adding potassium-rich foods to the diet, and avoiding strenuous exercises and physical exhaustion will help such patients to avoid hospitalization and lead a normal life.

\section{Conflicts of Interest}

None declared by the authors.

\section{Authors" contribution}

KD: Clinical management of the case, manuscript preparation

MB: Manuscript preparation, final draft editing

\section{References}

1. Fontaine B, Vale-Santos J, Jurkat-Rott K, Reboul J, Plassart E, Rime $C$ et al. Mapping of the hypokalaemic periodic paralysis (HypoPP) locus to chromosome 1q31-32 in three European families. Nature Genetics. 1994;6(3):267-272. [PubMed] DOI: 10.1038/ng0394-267

2. Wang W, Jiang L, Ye L, Zhu N, Su T, Guan L et al. Mutation screening in Chinese hypokalemic periodic paralysis patients. Mol Genet Metab. 2006; 87(4):359-363. [PubMed] DOI: 10.1016/j.ymgme.2005.10.020

3. Okinaka S, Shizume K, Uno S, Watanabe A, Irie M, Noguchi A et al. The association of periodic paralysis and hyperthyroidism in Japan. J Clin Endocrinol Metab. 1957 Dec;17(12):1454-9. [PubMed] DOI: 10.1210/jcem-17-12-1454 
4. Groome J, Moreau A, Delemotte L. Gating Pore Currents in Sodium Channels. Voltage-gated Sodium Channels. Handb Exp Pharmacol. 2018;246:371-399. [PubMed] DOI: 10.1007/164_2017_54

5. Jiang D, Gamal El-Din T, Ing C, Lu P, Pomès R, Zheng N et al. Structural basis for gating pore current in periodic paralysis.
Nature. 2018;557(7706):590-594 _ [PubMed] DOI: $10.1038 / s 41586-018-0120-4$

6. Kung AW. Thyrotoxic Periodic Paralysis: A Diagnostic Challenge. J Clin Endocrinol Metab. 2006 Jul;91(7):2490-5. [PubMed] DOI: 10.1210/jc.2006-0356 